\title{
REGNET: mining context-specific human transcription networks using composite genomic information
}

\author{
Sang-Mun Chi ${ }^{1+}$, Young-Kyo Seo ${ }^{2 \dagger}$, Young-Kyu Park ${ }^{3 \dagger}$, Sora Yoon ${ }^{2}$, Chan Young Park ${ }^{2}$, Yong Sung Kim³, \\ Seon-Young $\mathrm{Kim}^{3 *}$ and Dougu Nam ${ }^{2,4^{*}}$
}

\begin{abstract}
Background: Genome-wide expression profiles reflect the transcriptional networks specific to the given cell context. However, most statistical models try to estimate the average connectivity of the networks from a collection of gene expression data, and are unable to characterize the context-specific transcriptional regulations. We propose an approach for mining context-specific transcription networks from a large collection of gene expression fold-change profiles and composite gene-set information.

Results: Using a composite gene-set analysis method, we combine the information of transcription factor binding sites, Gene Ontology or pathway gene sets and gene expression fold-change profiles for a variety of cell conditions. We then collected all the significant patterns and constructed a database of context-specific transcription networks for human (REGNET). As a result, context-specific roles of transcription factors as well as their functional targets are readily explored. To validate the approach, nine predicted targets of E2F1 in HeLa cells were tested using chromatin immunoprecipitation assay. Among them, five (Gadd45b, Dusp6, MIl5, Bmp2 and E2f3) were successfully bound by E2F1. c-JUN and the EMT transcription networks were also validated from literature.
\end{abstract}

Conclusions: REGNET is a useful tool for exploring the ternary relationships among the transcription factors, their functional targets and the corresponding cell conditions. It is able to provide useful clues for novel cell-specific transcriptional regulations. The REGNET database is available at http://mgrc.kribb.re.kr/regnet.

Keywords: Composite gene-set analysis, Microarray, Transcription network, TFBS, Gene Ontology, KEGG

\section{Background}

Cells alter the process of transcriptional regulation so as to adjust to or drive changes in the cellular conditions between different stages of the cell cycle, stem cell differentiation or cancer development. Such changes in the transcriptional networks between different cell conditions are represented in the transcriptome data that are obtained using microarrays or high-throughput sequencing. However, elucidating the complex transcription networks (TNs) has been a daunting task in spite of the remarkable advances in both computational modeling and

\footnotetext{
*Correspondence: kimsy@kribb.re.kr; dougnam@unist.ac.kr

${ }^{\dagger}$ Equal contributors

${ }^{3}$ Medical Genomics Research Center, Korea Research Institute of Bioscience and Biotechnology, Daejeon, Republic of Korea

${ }^{2}$ School of Life Sciences, UNIST, Ulsan, Republic of Korea

Full list of author information is available at the end of the article
}

high-throughput experimental technologies [1-8]. One main reason for the difficulty is the dynamic nature of the networks: Transcription factors (TFs) not only regulate different targets depending on the cell conditions, but their effects on these targets can also change. Therefore, statistical models that estimate the average connectivity of the networks from a large collection of transcriptome data may confer limited accuracy.

Taking these issues into account, we have developed an approach for identifying the context-specific TNs from a large collection of gene expression fold-change profiles. Toward this end, we collected 2,482 paired (test/control) human microarray datasets encompassing a variety of cell conditions. Using a composite gene-set analysis method (ADGO) $[9,10]$, we combined the information of TF binding site (TFBS), Gene Ontology or KEGG pathway

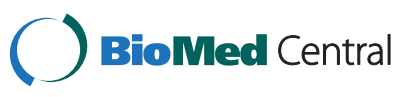


gene sets, and the gene expression fold-change profiles, and thereby tried to address the fundamental but largely open question of which TFs regulate which functions or pathways under differing cell conditions?

Since the majority of TFBSs are potentially false positives, we applied the following filtering criteria to identify reliable TNs as follows. (A) A TFBS gene set (genes that share a common TFBS in their promoters) is required to have a significant overlap with a functional gene set (genes that share a common annotation in GO or KEGG). (B) The genes that overlap, as a whole, should exhibit significant expression changes. (C) Such changes should be observed across many microarray conditions (e.g. ten or more). If the above three criteria are satisfied between a TFBS gene set and a functional gene set, we say the corresponding TF is associated with the functional gene set (Figure 1a), and the associated pairs as well as the corresponding conditions are output as context-specific TNs.

We collected all of the 12,149,291 significant triples (TF, functional gene set, condition; q-values for the two conditions (A) and (B) $<0.05)$ in order to construct a context-specific TN database, dubbed REGNET. It is used for exploring the ternary relationships among the TFs, their functional targets and the corresponding cell conditions (Figure 1b). Each TF or function (or pathway) name can be used as a query, and REGNET provides corresponding context-specific TNs. Using this database, we analyzed TNs for two TFs, E2F1 and cJUN. Many known target genes as well as corresponding microarray conditions were identified. REGNET also included many predictions for novel targets. For example, we selected nine candidate targets of E2F1 predicted for HeLa cells and tested them using a ChIP assay. Among them, five (Gadd45b, Dusp6, Mll5, $B m p 2$ and E2f3) were successfully bound by E2F1. We also validated the TNs for c-JUN and the 'epithelial to mesenchymal transition (EMT)' gene set from the literature. REGNET is available at http://mgrc.kribb.re. $\mathrm{kr} /$ regnet.

\section{Methods}

\section{Collection of data}

Human gene expression microarray datasets of the same platform 'HG-U133 Plus 2' were downloaded from 839 Gene Expression Omnibus [11] series. In each series dataset, we manually identified the test/control sample groups to collect log fold-change profiles. Many datasets contained multiple test groups, and hence 2,482 foldchange profiles were collected in total. Probe values that correspond to the same gene were averaged to yield gene-based fold-change profiles (20,361 genes). human TFBS gene sets were obtained from MSigDB [12]. For functional gene sets, the three categories of GO and KEGG pathways were used. GO gene sets were obtained from a gene product association file downloaded from a ftp site (http://www.geneontology.org/gene-associations/). Both the electronic and non-electronic annotations were used to maximize the coverage. KEGG gene sets were downloaded from MSigDB. All the offspring terms are again included in their parent terms. All the gene-sets with not less than 10 and not more than 500 genes are included in the analysis and the system.

\section{Identification of context-specific transcription networks}

We applied the following three filtering criteria to identify reliable TNs:

(A) Overlap significance: the significance of overlap for every pair of a TFBS gene set and functional gene set is assessed using the hypergeometric distribution. The genes that overlap, if significant (default: FDR q-value $<=0.02$ ), are regarded as candidate targets of the TF, and may be interpreted as the channel through which the TF regulates the function (or pathway). Such overlapping sets constitute the candidate TNs to be further examined throughout gene expression datasets.

(B) Expression significance: For each pair of TFBSfunctional gene sets that overlap significantly, another filtering criterion using a fold-change expression dataset is applied: The Z-statistic on the

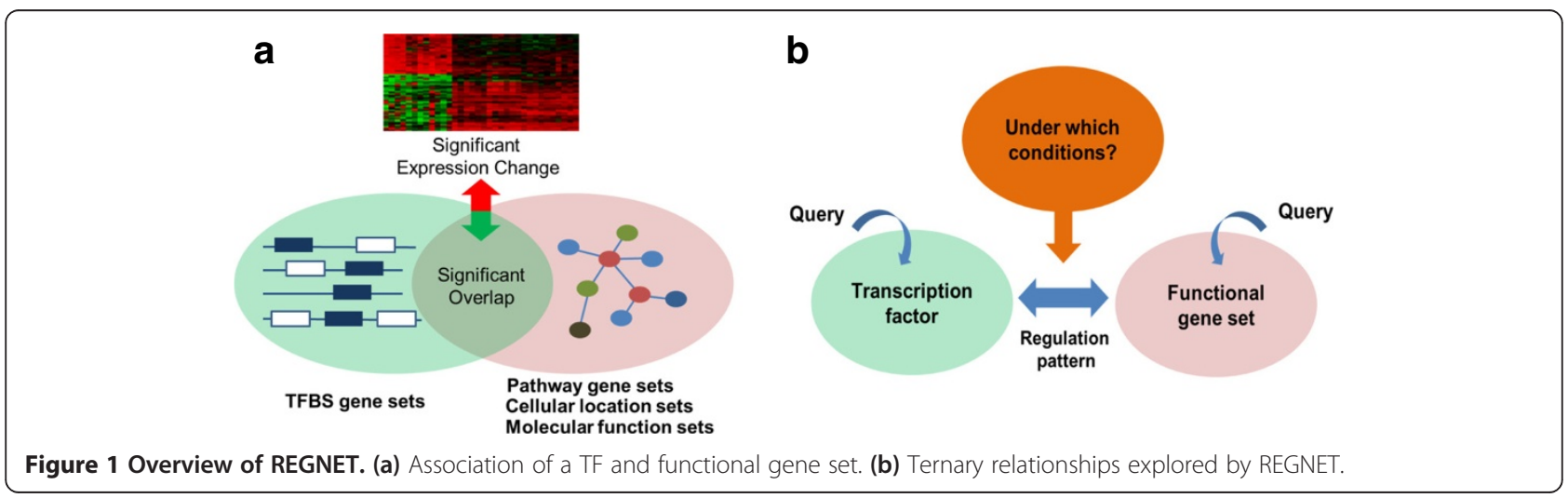


gene expression fold-change values is computed for each of the TFBS set, functional set and their overlapping set, respectively. If the FDR q-value of the overlapping set is less than or equal to 0.02 (default value) and the q-values for the two individual sets are larger than 0.05 (default value), we assume the corresponding TN is activated on the given microarray condition. In other words, expression changes in the overlapping genes should be pronounced compared to the two individual sets [9].

(C) Number of conditions: Only TNs that satisfy the above two criteria (A) and (B) across $k$ (default number $=10$ ) or more microarray conditions are regarded as reliable transcription patterns.

If all these criteria are satisfied between a TFBS gene set and functional gene set, the TF, candidate targets as well as the corresponding conditions are output as context-specific TN. Among the overlap set identified through (A) (C), we can further select individual candidate targets that show some high fold expression changes (e.g. \pm 1.5 of higher) across a number of conditions (e.g., more than $0.3 \times k$ conditions). All the threshold values including $\mathrm{q}$-values and $k$ can be changed by the user for more thorough exploration of the transcriptional regulations. Figure 2 summarizes the context-specific TNs queried by TF and functional gene set, respectively.

\section{Chromatin Immunoprecipitation (ChIP) assay for E2F1}

ChIP assays for E2F1 in HeLa cells were performed following the protocol represented at http://genomics.ucdavis. edu/farnham. HeLa cells were crosslinked for $10 \mathrm{~min}$ by adding formaldehyde to a final concentration of $1 \%$ with mild agitation. Crosslinking was stopped by the addition of glycine to a final concentration of $125 \mathrm{mM}$, and cells were washed three times with ice-cold PBS prior to harvesting by scraping of the plates. Chromatid were fragmented for $20 \mathrm{~min}(10 \mathrm{sec}$ on pulse and $20 \mathrm{sec}$ off pulse) to produce fragments $\sim 500 \mathrm{nt}$ in size using the Bioruptor sonicator (Diagenode). Antibody (SC251X; Santa Cruz Biotech) to E2F1 was used to pull down target chromatid from $1 \times 10^{8}$ cells. Genomic DNAs were isolated from proteinase $\mathrm{K}$-treated $\left(45^{\circ} \mathrm{C}\right.$ for $\left.1 \mathrm{~h}\right)$ samples and purified. ChIP samples were tested by PCR using positive and negative control primer sets shown in Additional file 1: Table S1. The quantitative real-time PCR for ChIP is described in Supplementary Material.

\section{Results}

\section{REGNET database}

REGNET is an intuitive and easy to use database developed for exploring context-specific TNs (http://mgrc. kribb.re.kr/regnet). REGNET accepts two kinds of queries, i.e. TF and function (or pathway, localization) names, respectively. It provides a webpage presenting all the TF names analyzed, so that investigators can simply choose the TF of their interest. Each TF name indicates a TFBS gene set to which the TF can bind. Because a TF can have different TFBSs, many of the TF names are represented multiple times. Among them, 'TF name)_all' is linked to the TNs that can be captured only by taking the union of all the TFBS sets of their common TF. For example, AHR_all links to the TNs that can be captured only by using the union of the four different TFBS sets (V\$AHRARNT_01, V\$AHRARNT_02, V\$AHR_01 and V\$AHR_Q5).

Each TF name is linked to a list of functional gene sets associated with the TF. Each line on the list is then linked to the corresponding target genes, descriptions for the TFBS and functional gene sets and the microarray
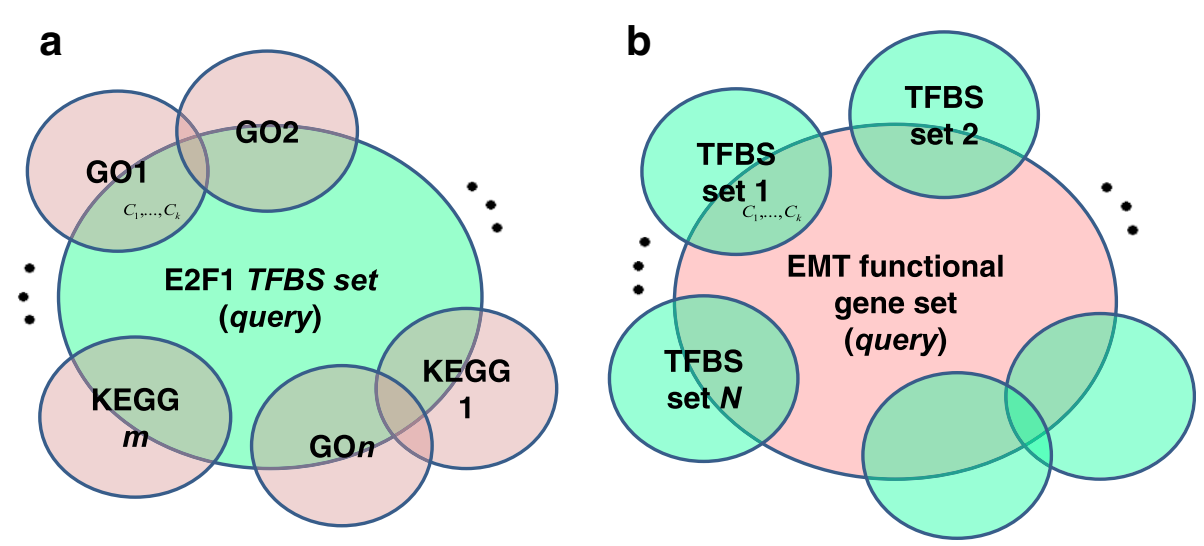

Figure 2 Context-specific transcription networks for the query of a TF or functional gene set. (a) For the query of a TF, multiple functional gene sets are associated. $C_{1}, C_{2}, \ldots, C_{k}$ represent the microarray conditions of the fold-change profiles for which the genes in the overlap parts significantly altered their expressions. The collection of the genes in the overlap parts are used for a global assessment. (b) For the query of a functional gene set, multiple TF's are associated. 
conditions under which the target genes exhibit significant changes in expression. The 'number of conditions' leads to a table of the corresponding test/control microarray conditions. At the bottom of the table, a heat map of the log fold-change values of the target genes is displayed, summarizing the information for the associated pair of the TF and functional gene set (Figure 3). From this heat map, investigators can easily identify candidate targets that exhibit large expression changes across a number of conditions. The fold-change values of the TF itself are also provided on the right-end column of the heat map, for the activity of certain TFs can be usefully inferred from their transcript levels.

When querying for a functional gene set, a keyword is required for searching. For example, if the investigators want to identify TNs related to the 'epithelial to mesenchymal transition' process, entering 'mesen' is sufficient. Then, the names of functional gene sets that contain 'mesen' are all displayed. From among them, investigators can choose the exact gene set of their interest, and then all the TFs associated with that functional gene set are listed.
As in the case of the TF query, each line is linked to detailed information on the target genes, TFBS sets and the queried gene set as well as the corresponding microarray conditions. More detailed information on the REGNET database may be seen from our web site.

\section{Analysis results for E2F1}

We used the human TFBS gene sets from the Molecular Signatures Database (MSigDB) [12]. The MSigDB provides five E2F1 TFBS sets. The predicted results for E2F1 are accessible by choosing ' $\mathrm{E}$ ' in the 'TF-based browsing' webpage. As expected, many cell-cycle related functional gene sets were found to be related to most of the TFBS gene sets. For example, the 'DNA replication (GO:0006260)' and 'G1/S transition of mitotic cell cycle (GO:0000082)' are associated with four of the five TFBS sets while the 'cell cycle (KEGG)' and 'cell cycle checkpoint (GO:0000075)' are associated with three. For each gene set, many conditions likely to activate cell cycle progression were observed, e.g. iPS or diverse cancer cell conditions.

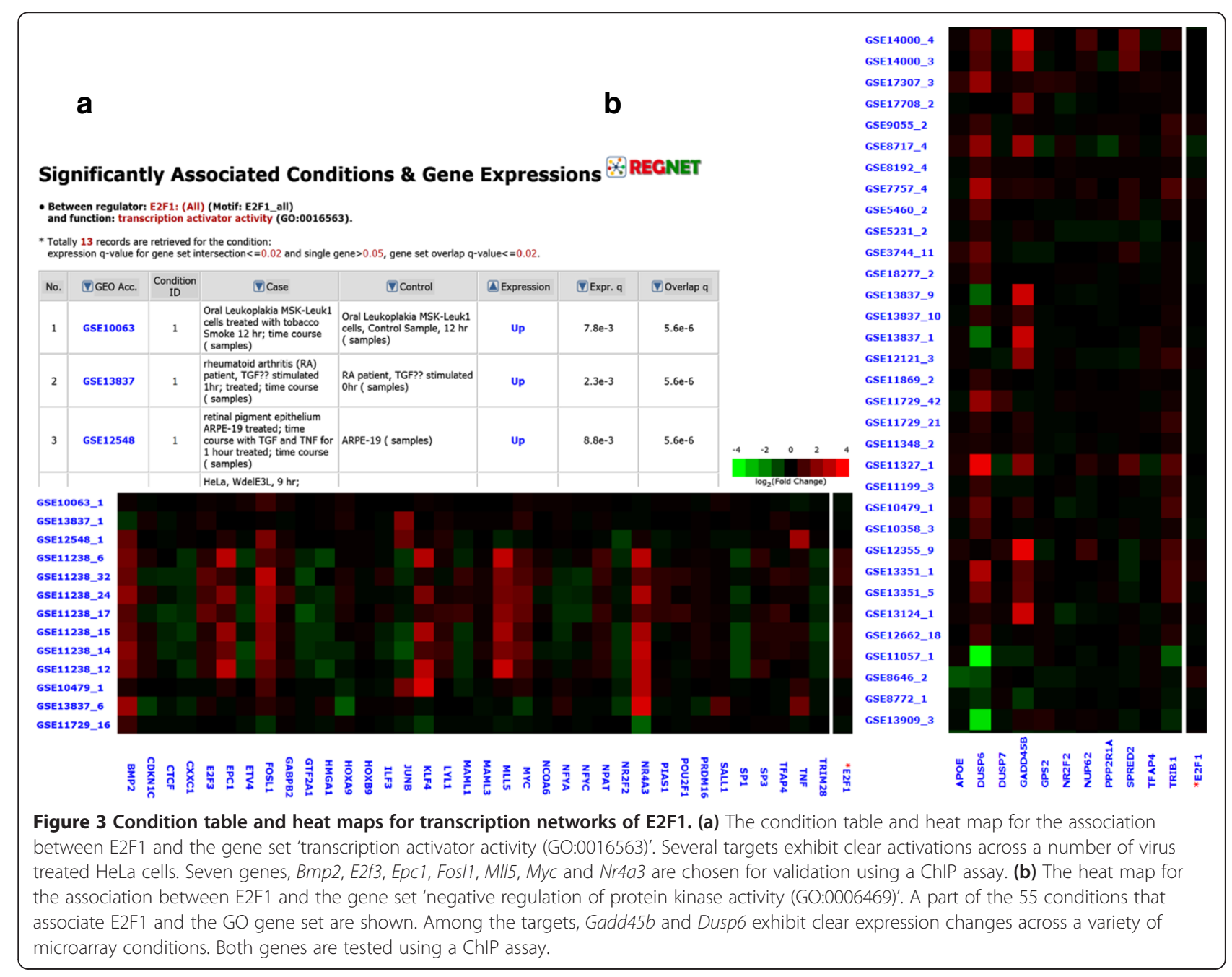


For the union of all the E2F1 TFBS gene sets (E2F1_all), the 'transcription activator activity (GO:0016563)' set is captured and several of the putative targets exhibit high expression fold-changes for a number of the HeLa cell conditions treated with vaccinia virus (GSE 11238): Bmp2, E2f3, Epc1, Fosl1, Mll5, Myc and Nr4a3 (Figure 3a). Another target set, 'negative regulation of protein kinase activity (GO:0006469)' is found to be associated with the E2F1_all set through 55 conditions. Among the targets, in particular, Dusp6 and Gadd45b exhibit high expression fold-changes across dozens of heterogeneous conditions (Figure 3b). Although these 55 conditions do not include the HeLa cells, it was found that both genes display more than two-fold increases in their expression levels in most of the virus-treated HeLa conditions (GSE 11238).

To validate the regulatory relationships, therefore, we performed a ChIP assay on HeLa cells, as described in Methods, for the nine candidate targets selected from the two associated sets 'transcription activator activity' and 'negative regulation of protein kinase activity'. To this end, we activated E2F1 via the CDK4-Rb-E2F1 pathway [13] as described in Additional file 1: Figure S1. We used Gapdh as a control. The ChIP results are shown in Figure 4. Among the targets tested, five exhibited positive results: The binding of E2F1 to Gadd45b, Dusp6 and Mll5 was pronounced with six or higher foldchanges compared to a control IgG, and the binding to $B m p 2$ and E2f3 exhibited approximate five and threefold increases, respectively.

\section{Analysis results for c-JUN}

The MSigDB provides eleven c-JUN TFBS sets. The predicted results for c-JUN are accessible by choosing ' $J$ ' in the TF-based browsing webpage. The first TFBS set named 'TGANTCA_V\$AP1_C' contains 866 genes, and 129 GO or KEGG gene sets are associated with this TFBS set as the result of satisfying the three filtering criteria
(A) (C). Among them, we identified two cell conditions for which c-JUN targets are most clearly activated. For example, the 'response to steroid hormone stimulus (GO:0048545)' gene set is associated with the c-JUN TFBS set for 56 microarray conditions. Among them, the HeLa cells treated with vaccinia virus (GSE 11238) clearly exhibits activation in five targets: Adm, F3, Fosl1, Il6 and Thbs1 (Additional file 1: Figure S2a). The latter four genes are known targets of c-JUN [14,15]. Thbs1 is known to be repressed by c-JUN in rat embryo fibroblasts [16], but it is also known to be activated by c-JUN in human hepatocarcinoma cell lines [15]. ADM is reported to induce the phosphorylation of c-JUN in glioblastoma cells [17], but is also a candidate target of c-JUN in our prediction. Because the known targets of c-JUN as well as Jun itself display high expression fold changes, we infer that viral infection to HeLa cells strongly increases the activity of c-JUN. In the 'pathways in cancer (KEGG)' gene set, we found another group of known c-JUN targets, i.e. Il6, Lamb3, Lamc2, Mmp1 and Mmp9 [14], that are strongly activated for the conditions of 'engineered human skin' (GSE 17539) [18] and 'low dose treatment of 5-aza-2-deoxycytidine on an non-small cell lung cancer cell line' (GSE 6695) [19] (Additional file 1: Figure S2b). If another TFBS set V\$AP1FJ_Q2 is chosen, the well-known c-JUN interacting partner Fos as well as Il6 are captured in the "pathways in cancer (KEGG)' gene set, and Fos exhibits a very strong co-expression pattern with Jun in many of the virus-treated HeLa cell conditions (Additional file 1: Figure S2c).

Summarizing the results for all the c-JUN TFBS gene sets, in total 156 functional gene sets are associated with at least one of the eleven TFBS gene sets. Of them, 52 functional gene sets (approximately 33\%) are associated with two or more. This suggests that c-JUN binds to a variety of different TFBSs in order to ensure the regulation of its target pathways. For example, 'pathways in

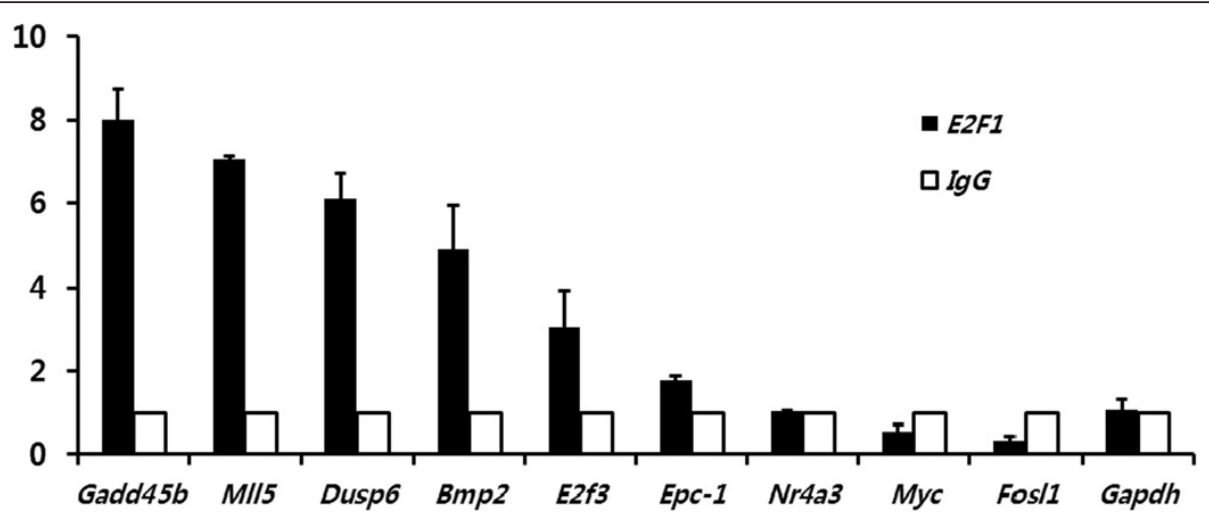

Figure 4 Chromatin IP experimental results for E2F1. The nine candidate targets of E2F1 were chosen for validation by gene-specific ChIP. The fold change (FC) is the fold increase of the signal from E2F1 antibody (black)-enriched chromatin relative to a control lgG (open). The negative control Gapdh exhibited no enrichment. 
cancer (KEGG)' is associated with eight different c-JUN TFBS gene sets, highlighting the role of c-JUN as a key factor of cancer progression. The representative gene sets associated with three or more c-JUN TFBS sets are shown in Table 1.

\section{Analysis results for the EMT gene set}

We now illustrate how to analyze a functional gene set using REGNET. Entering the keyword 'mesen' from the 'Keyword Search' page, nine similar functional gene sets were presented. Among them, we chose the first one, i.e. the 'epithelial to mesenchymal transition (GO:0001837)'. This set is associated with eight TFs (MAZ, FOXO4, PITX2, SP1, NFAT, PAX4, ZEB1 and LEF1). Except for MAZ and PAX4, each TF is reported to regulate EMT process. We then investigated the conditions under which each TF might regulate the EMT process. Many putative conditions that are likely to trigger EMT were observed. For example, PCDH24-expressing HCT116 cells shown to abolish tumor formation in vivo have 2000 differentially expressed genes. These expression changes have been confirmed to be quite similar to EMT via further proteomics analysis using 2-DE/MS (GSE10650). Our predictions on EMT suggest MAZ as a candidate TF to regulate EMT in the HCT116 cells. Among the candidate targets of MAZ in the EMT gene set, Bmp7, Col1a1, Sfrp2, Wnt2 and Wnt5a exhibit two or higher expression fold-changes. Interestingly, four of the targets except for Sfrp2 were also captured among the PAX4 results for the same cell condition. This indicates PAX4 and MAZ possibly cooperate on their four common targets to regulate EMT in the HCT cells. For another example, the embroid body cells (GSE9196) the differentiation of which is known to accompany EMT
[20] also exhibit clear activation in many of the MAZ targets (Additional file 1: Figure S3a).

Among the conditions, HUVECs treated with TNFalpha are captured by five of the eight TF results (Additional file 1: Figure S3b-e). By mapping each TF to the targets that exhibit two or higher fold-changes, the EMT transcription networks in HUVECs treated with TNF-alpha were constructed that comprised five TFs and six target genes (Figure 5). TNF-alpha is a key cytokine involved in inflammation and cancer, and is known to induce EMT [21]. Because HUVECs do not contain epithelial cells, such EMT signals may indicate 'endothelial' to mesenchymal transitions.

Although each regulatory relationship in the networks needs to be validated, it is noteworthy that the predicted edges of the networks were evenly distributed among the five TFs. This implies that the EMT in HUVECs is processed by a tight cooperation of these regulators. Some of the reports for the predicted regulatory relationships are provided in Additional file 1: Table S2.

\section{A global assessment of REGNET}

Because the predicted TNs in REGNET are contextspecific (the targets or effects of a TF can change depending on the cell conditions), reliable gold-standard TNs for a global assessment are rarely available. For example, each ChIP-seq or ChIP-chip dataset is also obtained under a specific cell condition and may not serve as a general gold-standard set. Therefore, instead of considering individual cell conditions, we merged all the context-specific targets of a TF for our prediction set (the union of the intersection parts in Figure 2a), and merged multiple ChIP-seq or ChIP-chip data for goldstandard targets of a TF. Among the prediction set, we

Table 1 Functional gene sets associated with c-JUN

\begin{tabular}{lll}
\hline Functional gene set codes & Functional gene set names & \#TFBS sets* \\
\hline KEGG PATHWAYS IN CANCER (KEGG) & Pathways in cancer & 8 \\
GO:0000165 (GOBP) & MAPKKK cascade & 6 \\
GO:0043086 (GOBP) & Negative regulation of catalytic activity \\
GO:0031012 (GOCC) & Extracellular matrix \\
GO:0045859 (GOBP) & Regulation of protein kinase activity \\
KEGG MAPK SIGNALING PATHWAY (KEGG) & MAPK signaling pathway \\
GO:0001501 (GOBP) & Skeletal system development \\
GO:0001525 (GOBP) & Angiogenesis \\
GO:0007409 (GOBP) & Axonogenesis \\
GO:0018193 (GOBP) & Peptidyl-amino acid modification \\
GO:0030334 (GOBP) & Regulation of cell migration \\
GO:0032270 (GOBP) & Positive regulation of cellular protein metabolic process \\
GO:0043066 (GOBP) & Negative regulation of apoptosis \\
GO:0048812 (GOBP) & Neuron projection morphogenesis & 3 \\
\hline
\end{tabular}

*The number of c-JUN TFBS gene sets associated with each functional gene set. 


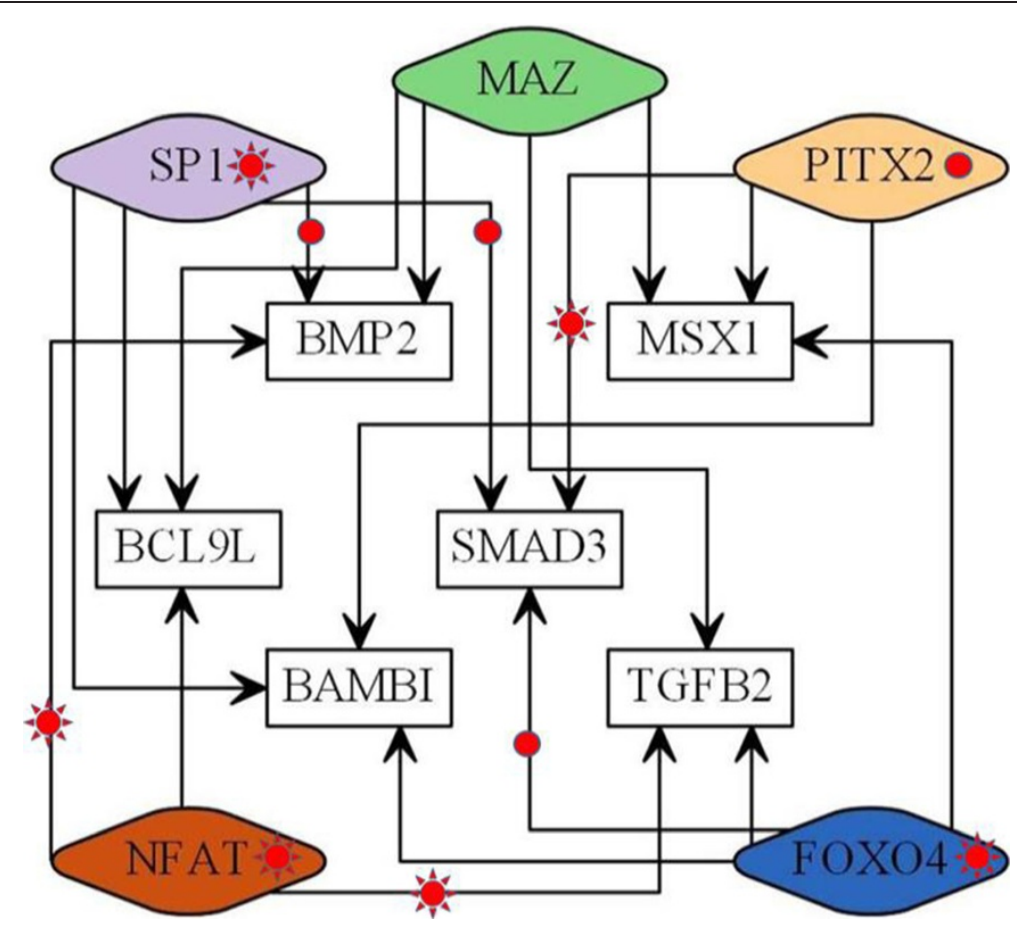

Figure 5 EMT transcription networks in HUVECs inferred from REGNET. The sun symbols indicate the corresponding TFs or TF-target relationships have strong supports from the literature. The circle symbols indicate weak supports from the literature. The evidences from the literature are all summarized in Additional file 1: Table S2. The graph is depicted using the Graphviz software.

further selected candidate targets that exhibited \pm 1.5 or higher fold expression changes across $0.3 \times k$ conditions. We validated the predicted targets of E2F1, JUN and TP53. Because these TFs have multiple known binding sites in MSigDB, we merged all the corresponding targets for each TF. The targets reported from ChIP data are downloaded from ChEA curated database [22]. Three, one and six ChIP datasets were available for E2F1, JUN and TP53, respectively and corresponding targets were merged for each TF. These targets with known TFBS were further selected for true positive gold standard sets.

Among the 564 genes with an E2F1 binding site, 65 were the predicted as context-specific targets. Among the 270 true positive set, 39 were included in the 65 predicted set. This corresponds to specificity 0.9116 and sensitivity 0.1444 with a significant $p$-value 0.0256 against a random prediction (hypergeometric distribution). The true positive set used here is obtained from a collection of highthroughput ChIP datasets and is missing the three targets Dusp6, Mll5 and Gadd45b, while these targets were identified from our ChIP assay. If we include these three in the positive set, we have an improved sensitivity 0.1538 with a significant p-value 0.0039. Unfortunately, JUN had only one ChIP data in ChEA database. Based on this single ChIP dataset, we had specificity 0.7853 , sensitivity 0.2593 but with a less significant p-value 0.1734 . Lastly, TP53 had six ChIP datasets. Among the 426 genes with an P53 binding site, 142 were true positives and the 50 predicted targets included 25 true positives. This corresponds to specificity 0.9120 , sensitivity 0.1761 and a significant p-value 0.0071 .

In summary, REGNET provides highly specific predictions for context-specific transcriptional regulations. Though less sensitive, they still contain a number of novel regulatory relationships as shown in our ChIP assay and the EMT networks.

\section{Assessment for extended GO and KEGG annotations}

Because many genes are still unannotated in $G O$ and KEGG, transcriptional regulations for such genes are not covered by REGNET. To ameliorate the problem, we extended GO and KEGG annotations using proteinprotein interaction networks [23], and tested how the extended annotations affect the predictions in REGNET. The annotations are extended as follows: For each gene $g_{i}$ (protein), we identified the set of interacting proteins $L_{i}$ from the BioGrid database [24]. For each functional gene set (GO or KEGG annotation), say $F_{j}$, we assessed the significance of overlap between $L_{i}$ and $F_{j}$ using hypergeometric distribution. If an overlap $\mathrm{p}$-value $\leq \mathrm{p}_{0}$, we assign the annotation $F_{j}$ to the gene $g_{i}$.

In the original GO and KEGG annotations, 2,409 GO biological process terms (GO-BP), 369 GO cellular component terms (GO-CC), $186 \mathrm{GO}$ molecular function terms (GO-MF) and $666 \mathrm{KEGG}$ terms are used to 
annotate genes 164,562, 26,270, 12,875 and 41,063 times respectively. When a threshold $\mathrm{p}_{0}=10 E_{-4}$ is applied, GO-BP, GO-CC, GO-MF and KEGG terms are used 233,878, 37,455, 23,370 and 54,142 times exhibiting overall $29.8 \%$ increase. Using these extended annotations, we regenerated significant patterns in REGNET and extracted context-specific targets of E2F1 and TP53, respectively. With the original annotations, 65 and 50 context-specific targets were identified for E2F1 and TP53, but the extended annotations increased these numbers to 183 and 71, respectively. The extended predictions, as expected, exhibited somewhat reduced specificities 0.7313 and 0.8627 , and increased sensitivities 0.3852 and 0.2253 for E2F1 and TP53, respectively.

Here, we illustrated how current annotations can be extended using protein interaction data and how such extension could affect the predictions in REGNET, but such extensions are not included in the current REGNET system.

\section{Discussion and conclusions}

For a given TF or a functional gene set, REGNET provides the associated TNs as well as the corresponding conditions. From the heat map provided for each of the associated pairs of a TF and functional gene set, investigators can select putative targets that exhibit large expression changes across a number of different conditions. We note that the transcription networks inferred in our approach have multiple lines of evidences and a substantial portion of such predictions are highly likely true as validated by reports in the literature and the ChIP assay in the finding reported here.

For a global validation, we pooled targets from multiple ChIP datasets and pooled context-specific predictions for each of three TFs. As expected, predictions in REGNET were highly specific and less sensitive. For example, REGNET provides 65 context-specific targets of E2F1, 42 of which were actually bound by the TF. More importantly, REGNET suggests the cell conditions in which such regulation patterns occur. Despite the high specificity, predictions by REGNET included many novel targets providing useful clues for unraveling cellular processes.

Because many microarray datasets have only a small number of samples, we applied a gene randomizing gene set analysis method [25,26] to assess the overall expression changes in each gene set. Most gene-randomizing methods, however, suffer from increased false-positives [26]. To reduce false positives, we applied an auxiliary filter that requires a significant regulation pattern to be observed across a number of different conditions.

Specifically, REGNET deploys a composite gene-set analysis method $[9,10]$ to combine three sources of genomic information: TFBS gene set, functional gene set and gene expression fold-change profile. As a result, three- dimensional information among the TFs, their functional targets and cell conditions are readily explored. Most other approaches including DAVID [27], the Molecular Concept Map [28] and ConceptGen [29] investigate the 'binary' relationships between a TFBS gene set and another gene list. One may apply the overlap of gene-sets once more to investigate such three-dimensional relationships, but the repeated use of gene-set overlaps may cause a serious loss of power due to the use of threshold values [26,30].

Because the activity of a TF is estimated by the overall pattern of expression change in its target genes, REGNET is most useful for finding relatively strong transcriptional activities in which a TF regulates multiple target genes simultaneously. On the other hand, specific regulation of one or two targets that have only small expression changes might be missed in this approach. Another limitation is that by using the TFBS information instead of genome-wide ChIP data, which are mostly unavailable, we only consider the sequence-specific transcriptional regulation. Furthermore, many gene functions are still unknown and remain unannotated in GO or KEGG. We here demonstrated a method to extend annotation gene-sets using protein interaction data and its effect on the performance of REGNET. More reliable extension of gene annotation may be possible by integrating diverse types of genomic data and network information which may help identifying missing transcriptional associations in REGNET [31-33].

Over the last decade, more than a million gene expression microarray datasets have been deposited in public databases $[11,34,35]$, covering virtually all of the cell conditions of interest including a broad range of tissues, diseases, development and treatments. Most of them are made up of test and control samples, and their fold-change values provide information on the TNs specific to each condition. For this reason, we have collected the foldchange profiles for a diverse array of cell conditions in order to identify context-specific TNs. We have collected 2,482 human expression fold-change profiles in our current database. As this number increases, it is expected that more specific TNs are newly identified. Moreover, developing a similar system for other species would be valuable for the purpose of comparative analysis.

\section{Additional file}

Additional file 1: The description on ChIP assay and EMT networks. Figure S1. ChIP assay design. Figure S2. Heat maps associated with c-JUN. Figure S3. EMT transcription networks by five TFs. Table S1.

Oligos used for ChIP assays. Table S2. Evidences of the EMT transcription networks from literature.

\section{Abbreviations}

TF: Transcription factor; TFBS: Transcription factor binding site;

TN: Transcription network; ChIP: Chromatin immunoprecipitation; GO: Gene 
ontology; KEGG: Kyoto encyclopedia of genes and genomes; MSigDB: Molecular signature database; EMT: Epithelial to mesenchymal transition.

\section{Competing interests}

The authors declare that they have no competing interests.

\section{Authors' contributions}

SMC and YKP developed the database, YKS did ChIP assay and CYP confirmed the result. SY aided literature study. SYK, DN and YSK COsupervised the development of database. SYK collected microarray datasets. DN wrote the manuscript. All authors read and approved the final manuscript.

\section{Acknowledgements}

This work was supported by Basic Science Research Program through a National Research Foundation (NRF) grants funded by the Korean government (MEST) (No. 2012-0003589 and No. 2011-0020163) to DN, and by genomics (2012M3A9D1054670) program of the National Research Foundation of Korea funded by the Ministry of Education, Science and Technology (MEST) to SYK.

\section{Author details}

${ }^{1}$ School of Computer Science and Engineering, Kyungsung University, Busan, Republic of Korea. ${ }^{2}$ School of Life Sciences, UNIST, UIsan, Republic of Korea. ${ }^{3}$ Medical Genomics Research Center, Korea Research Institute of Bioscience and Biotechnology, Daejeon, Republic of Korea. ${ }^{4}$ Division of Mathematical Sciences, UNIST, Ulsan, Republic of Korea.

Received: 22 August 2013 Accepted: 27 May 2014

Published: 9 June 2014

\section{References}

1. Bansal M, Belcastro V, Ambesi-Impiombato A, di Bernardo D: How to infer gene networks from expression profiles. Mol Syst Biol 2007, 3:78.

2. Harbison CT, Gordon DB, Lee TI, Rinaldi NJ, Macisaac KD, Danford TW, Hannett NM, Tagne JB, Reynolds DB, Yoo J, Jennings EG, Zeitlinger J, Pokholok DK, Kellis M, Rolfe PA, Takusagawa KT, Lander ES, Gifford DK, Fraenkel E, Young RA: Transcriptional regulatory code of a eukaryotic genome. Nature 2004, 431(7004):99-104.

3. Chen $X, X u H$, Yuan P, Fang F, Huss M, Vega VB, Wong E, Orlov YL, Zhang W, Jiang J, Loh YH, Yeo HC, Yeo ZX, Narang V, Govindarajan KR, Leong B, Shahab A, Ruan Y, Bourque G, Sung WK, Clarke ND, Wei CL, Ng HH: Integration of external signaling pathways with the core transcriptional network in embryonic stem cells. Cell 2008, 133(6):1106-1117.

4. He F, Balling R, Zeng AP: Reverse engineering and verification of gene networks: principles, assumptions, and limitations of present methods and future perspectives. J Biotechnol 2009, 144(3):190-203.

5. Wingender E, Chen X, Fricke E, Geffers R, Hehl R, Liebich I, Krull M, Matys V, Michael H, Ohnhauser R, Prüss M, Schacherer F, Thiele S, Urbach S: The TRANSFAC system on gene expression regulation. Nucleic Acids Res 2001, 29(1):281-283.

6. Wasserman WW, Sandelin A: Applied bioinformatics for the identification of regulatory elements. Nat Rev Genet 2004, 5(4):276-287.

7. Kato M, Hata N, Banerjee N, Futcher B, Zhang MQ: Identifying combinatorial regulation of transcription factors and binding motifs. Genome Biol 2004, 5(8):R56.

8. Gao F, Foat BC, Bussemaker HJ: Defining transcriptional networks through integrative modeling of mRNA expression and transcription factor binding data. BMC Bioinformatics 2004, 5:31.

9. Chi SM, Kim J, Kim SY, Nam D: ADGO 2.0: interpreting microarray data and list of genes using composite annotations. Nucleic Acids Res 2011, 39:W302-306. Web Server issue.

10. Nam D, Kim SB, Kim SK, Yang S, Kim SY, Chu IS: ADGO: analysis of differentially expressed gene sets using composite $\mathrm{GO}$ annotation. Bioinformatics 2006, 22(18):2249-2253.

11. Barrett $T$, Wilhite SE, Ledoux P, Evangelista C, Kim IF, Tomashevsky M, Marshall KA, Phillippy KH, Sherman PM, Holko M, Yefanov A, Lee H, Zhang N, Robertson CL, Serova N, Davis S, Soboleva A: NCBI GEO: archive for functional genomics data sets-update. Nucleic Acids Res 2013,

41(Database issue):D991-995.
12. Liberzon A, Subramanian A, Pinchback R, Thorvaldsdottir $H$, Tamayo $P$, Mesirov JP: Molecular signatures database (MSigDB) 3.0. Bioinformatics 2011, 27(12):1739-1740.

13. Satyanarayana A, Kaldis P: Mammalian cell-cycle regulation: several Cdks, numerous cyclins and diverse compensatory mechanisms. Oncogene 2009, 28(33):2925-2939.

14. Lee JS, Heo J, Libbrecht L, Chu IS, Kaposi-Novak P, Calvisi DF, Mikaelyan A, Roberts LR, Demetris AJ, Sun Z, Nevens F, Roskams T, Thorgeirsson SS: A novel prognostic subtype of human hepatocellular carcinoma derived from hepatic progenitor cells. Nat Med 2006, 12(4):410-416.

15. Kim SA, Um SJ, Kang JH, Hong KJ: Expression of thrombospondin-1 in human hepatocarcinoma cell lines and its regulation by transcription factor Jun/AP-1. Mol Cell Biochem 2001, 216(1-2):21-29.

16. Mettouchi A, Cabon F, Montreau N, Vernier P, Mercier G, Blangy D, Tricoire $H$, Vigier $P$, Binetruy B: SPARC and thrombospondin genes are repressed by the c-jun oncogene in rat embryo fibroblasts. EMBO J 1994, 13(23):5668-5678.

17. Ouafik L, Berenguer-Daize C, Berthois Y: Adrenomedullin promotes cell cycle transit and up-regulates cyclin D1 protein level in human glioblastoma cells through the activation of c-Jun/JNK/AP-1 signal transduction pathway. Cell Signal 2009, 21(4):597-608.

18. Klingenberg JM, McFarland KL, Friedman AJ, Boyce ST, Aronow BJ, Supp DM: Engineered human skin substitutes undergo large-scale genomic reprogramming and normal skin-like maturation after transplantation to athymic mice. J Invest Dermatol 2010, 130(2):587-601.

19. Shames DS, Girard L, Gao B, Sato M, Lewis CM, Shivapurkar N, Jiang A, Perou CM, Kim YH, Pollack JR, Fong KM, Lam CL, Wong M, Shyr Y, Nanda R, Olopade Ol, Gerald W, Euhus DM, Shay JW, Gazdar AF, Minna JD: A genome-wide screen for promoter methylation in lung cancer identifies novel methylation markers for multiple malignancies. PLOS Med 2006, 3(12):e486.

20. ten Berge D, Koole W, Fuerer C, Fish M, Eroglu E, Nusse R: Wnt signaling mediates self-organization and axis formation in embryoid bodies. Cell Stem Cell 2008, 3(5):508-518.

21. Wu Y, Zhou BP: TNF-alpha/NF-kappaB/Snail pathway in cancer cell migration and invasion. Br J Cancer 2010, 102(4):639-644.

22. Lachmann A, Xu H, Krishnan J, Berger SI, Mazloom AR, Ma'ayan A: ChEA: transcription factor regulation inferred from integrating genome-wide ChIP-X experiments. Bioinformatics 2010, 26(19):2438-2444.

23. Jansen R, Yu H, Greenbaum D, Kluger Y, Krogan NJ, Chung S, Emili A, Snyder M, Greenblatt JF, Gerstein M: A Bayesian networks approach for predicting protein-protein interactions from genomic data. Science 2003, 302(5644):449-453.

24. Chatr-Aryamontri A, Breitkreutz BJ, Heinicke S, Boucher L, Winter A, Stark C, Nixon J, Ramage L, Kolas N, O'Donnell L, Reguly T, Breitkreutz A, Sellam A, Chen D, Chang C, Rust J, Livstone M, Oughtred R, Dolinski K, Tyers M: The BioGRID interaction database: 2013 update. Nucleic Acids Res 2013, 41(Database issue):D816-823.

25. Kim SY, Volsky DJ: PAGE: parametric analysis of gene set enrichment. BMC Bioinformatics 2005, 6:144.

26. Nam D, Kim SY: Gene-set approach for expression pattern analysis. Brief Bioinform 2008, 9(3):189-197.

27. da Huang W, Sherman BT, Lempicki RA: Systematic and integrative analysis of large gene lists using DAVID bioinformatics resources. Nat Protoc 2009, 4(1):44-57.

28. Rhodes DR, Kalyana-Sundaram S, Tomlins SA, Mahavisno V, Kasper N Varambally R, Barrette TR, Ghosh D, Varambally S, Chinnaiyan AM: Molecular concepts analysis links tumors, pathways, mechanisms, and drugs. Neoplasia 2007, 9(5):443-454.

29. Sartor MA, Mahavisno V, Keshamouni VG, Cavalcoli J, Wright Z, Karnovsky A, Kuick R, Jagadish HV, Mirel B, Weymouth T, Athey B, Omenn GS: ConceptGen: a gene set enrichment and gene set relation mapping tool. Bioinformatics 2010, 26(4):456-463.

30. Mootha VK, Lindgren CM, Eriksson KF, Subramanian A, Sihag S, Lehar J, Puigserver P, Carlsson E, Ridderstrale M, Laurila E, Houstis N, Daly MJ, Patterson N, Mesirov JP, Golub TR, Tamayo P, Spiegelman B, Lander ES, Hirschhorn JN, Altshuler D, Groop LC: PGC-1alpha-responsive genes involved in oxidative phosphorylation are coordinately downregulated in human diabetes. Nat Genet 2003, 34(3):267-273.

31. Wang PI, Hwang S, Kincaid RP, Sullivan CS, Lee I, Marcotte EM: RIDDLE: reflective diffusion and local extension reveal functional associations for 
unannotated gene sets via proximity in a gene network. Genome Biol 2012, 13(12):R125.

32. Dutkowski J, Kramer M, Surma MA, Balakrishnan R, Cherry JM, Krogan NJ, Ideker T: A gene ontology inferred from molecular networks. Nat Biotechnol 2013, 31(1):38-45.

33. Chua HN, Sung WK, Wong L: An efficient strategy for extensive integration of diverse biological data for protein function prediction. Bioinformatics 2007, 23(24):3364-3373.

34. Rustici G, Kolesnikov N, Brandizi M, Burdett T, Dylag M, Emam I, Farne A, Hastings E, Ison J, Keays M, Kurbatova N, Malone J, Mani R, Mupo A, Pedro Pereira R, Pilicheva E, Rung J, Sharma A, Tang YA, Ternent T, Tikhonov A, Welter D, Williams E, Brazma A, Parkinson H, Sarkans U: ArrayExpress update-trends in database growth and links to data analysis tools. Nucleic Acids Res 2013, 41(Database issue):D987-990.

35. Demeter J, Beauheim C, Gollub J, Hernandez-Boussard T, Jin H, Maier D, Matese JC, Nitzberg M, Wymore F, Zachariah ZK, Brown PO, Sherlock G, Ball CA: The Stanford microarray database: implementation of new analysis tools and open source release of software. Nucleic Acids Res 2007, 35(Database issue):D766-770.

doi:10.1186/1471-2164-15-450

Cite this article as: Chi et al:: REGNET: mining context-specific human transcription networks using composite genomic information. BMC Genomics 2014 15:450.

\section{Submit your next manuscript to BioMed Central and take full advantage of:}

- Convenient online submission

- Thorough peer review

- No space constraints or color figure charges

- Immediate publication on acceptance

- Inclusion in PubMed, CAS, Scopus and Google Scholar

- Research which is freely available for redistribution 